\title{
Total Skin Electron Beam Radiation Therapy
}

National Cancer Institute

\section{Source}

National Cancer Institute. Total Skin Electron Beam Radiation Therapy. NCI Thesaurus.

Code C93341.

Electron beam radiation therapy that targets the entire skin. 